\title{
SENSIBILIDAD AL ASCO: CONCEPTO Y RELACIÓN CON LOS MIEDOS Y LOS TRASTORNOS DE ANSIEDAD
}

\author{
Bonifacio Sandín, Paloma Chorot, Miguel A. Santed, \\ Rosa M. VALIENTE Y MARGARITA OLMEDO
}

Facultad de Psicología, Universidad Nacional de Educación a Distancia, Madrid

\begin{abstract}
Resumen: Aunque en los últimos años se ha producido un creciente interés por la investigación sobre el papel de la sensibilidad al asco en psicopatología, y en particular en los trastornos de ansiedad, la evidencia no es concluyente y presenta diversas controversias. En este trabajo llevamos a cabo una revisión sobre la relación entre la sensibilidad al asco y diversos aspectos relacionados con la ansiedad, y los trastornos de ansiedad. Tras clarificar el significado del concepto del asco como emoción básica, se analiza su relación con síntomas y trastornos relativos a ciertas fobias específicas (i.e., miedos y/o fobias a los «animales repugnantes» - incluidas las fobias a las arañas — y fobias del tipo sangre-inyecciones-daño) y al trastorno obsesivo-compulsivo. Finalmente se discute el papel psicopatológico del asco y algunos aspectos emergentes, tales como la implicación de las distintas dimensiones del asco, la separación entre propensión y sensibilidad al asco, y la función y ubicación de la propensión/sensibilidad del asco como factor de vulnerabilidad en el marco del modelo jerárquico de los miedos y las fobias (S. Taylor, 1998; B. Sandín \& P. Chorot, 1999).
\end{abstract}

Palabras clave: Sensibilidad al asco, propensión al asco, miedos, fobias, fobia a las arañas, fobia a la sangre-inyecciones-daño, trastorno obsesivo-compulsivo, miedo a la contaminación, trastornos de ansiedad.

\section{Disgust sensitivy: Concept and relationship with fears and anxiety disorders}

\begin{abstract}
There is an increasing research interest on the role of disgust in psychopathology. Although in the last few years one major area of research has investigated the role of disgust in anxiety disorders and anxiety disorder symptoms, this line of research is not without controversy. In this work we accomplish an evaluative review on relationship between disgust sensitivity and some anxiety symptoms, fears and anxiety disorders. Following a clarification of the concept of disgust as a basic emotion, we examine it association with symptoms and disorders related to specific phobias (i.e., phobias to «repulsive animals» — including spider phobias — and blood-injection-injury phobias) and obsessive-compulsive disorder. Finally, we discuss some emerging issues such as the role of disgust domains, the separation between propensity and sensitivity, and the position of disgust propensity/sensitivity as a vulnerability factor in the hierarchic model of fears (S. Taylor, 1998; B. Sandín \& P. Chorot, 1999).
\end{abstract}

Keywords: Disgust sensitivity, disgust propensity, fears, phobias, spider phobia, blood-injectioninjury phobia, obsessive-compulsive disorder, contamination fear, anxiety disorders.

\section{INTRODUCCIÓN: ASCO Y SENSIBILIDAD AL ASCO}

El asco es una emoción básica con componentes cognitivos, fisiológicos y conductuales distintivos que actúa para prevenir la contami-

Recibido 4 abril 2008; aceptado 5 mayo 2008.

Correspondencia: Bonifacio Sandín, Universidad Nacional de Educación a Distancia (UNED), Facultad de Psicología, Juan del Rosal 10, 28040 Madrid.

Correo-e: bsandin@psi.uned.es nación y la enfermedad (Rozin y Fallon, 1987). Ha sido definido como la repugnancia ante la posible incorporación oral de algún objeto repelente; los objetos repelentes son contaminantes (si tienen contacto con los alimentos hacen que éstos se conviertan en inaceptables). Los objetos calificados como asquerosos generalmente son animales (o partes de estos) o productos de sus cuerpos (orina, etc.) (Sandín, 1997). En el lenguaje anglosajón, disgust (asco) significa mal gusto o sabor ( «bad taste»), lo cual denota 
su asociación primigenia (evolutivo adaptativa) con el rechazo de la ingesta oral de alimentos, protegiendo de este modo al organismo de la incorporación oral de posibles sustancias peligrosas y promoviendo la evitación de la enfermedad. En general, la mayor parte de los autores han relacionado el asco con la comida y el sabor (incorporación oral de sustancias), excepto Freud que lo relacionó esencialmente con el sexo (la reacción de asco serviría, según este autor, para restringir las fantasías sexuales a las prácticas sexuales aceptables socialmente). Sin embargo, como han señalado Haidt, McCauley y Rozin (1994), la reacción de asco no se basa primariamente en el sabor, sino más bien en el conocimiento sobre la naturaleza y origen de un alimento potencial; las cosas que saben mal no poseen necesariamente la propiedad de ser contaminantes. El asco se ha entendido también como una reacción primaria ante la intimidad no deseada.

Se ha sugerido que, al igual que ocurre con el miedo, el asco podría desempeñar una función claramente adaptativa (Matchett y Davey, 1991). De modo similar al miedo, el asco parece poseer un papel protector al motivar la conducta de evitación ante estímulos o situaciones potencialmente generadoras de enfermedad o contaminación. Por tanto, similarmente a lo que ocurre con el miedo, las respuestas de asco podrían ser entendidas como respuestas emocionales preparadas filogenéticamente. En cierto modo, ambos tipos de respuestas preparadas (i.e., el miedo y el asco) podrían actuar sinérgicamente, al menos en ciertas circunstancias. Así mismo, el asco podría jugar un papel similar al del miedo en el mantenimiento de la jerarquía social, mediante el desprecio hacia individuos de diferente clase social, y reforzado las conductas sexuales apropiadas (p.ej., el asco asociado a la violación del tabú del incesto) (Woody y Teachman, 2000). Este paralelismo en propiedades protectoras y comunicativas entre el miedo y el asco podría tener importantes implicaciones para comprender el hecho de que a veces un mismo estímulo (p.ej., la vista de la sangre) pueda generar ambas formas de emoción.

Aparte de la atención que haya suscitado el estudio del asco desde su consideración como emoción básica, recientemente se ha produci- do un particular interés por el estudio de la sensibilidad al asco desde el punto de vista psicopatológico. La sensibilidad al asco ha sido definida en términos de diferencias individuales en la predisposición a experimentar la emoción de asco (p.ej., Berle y Phillips, 2006; Haidt et al., 1994; Olatunji y Sawchuk, 2005; Rozin, Haidt y McCauley, 2000; Woody y Teachman, 2000; Woody y Tolin, 2002). Aunque algunos autores (p.ej., van Overveld, de Jong, Peters, Cavanagh y Davey, 2006) han indicado que este concepto de sensibilidad al asco consiste más bien en propensión que en sensibilidad, como norma, salvo que se indique lo contrario, adoptaremos el concepto indicado arriba para referirnos a la sensibilidad al asco de modo genérico (ya que este es el concepto que ha prevalecido hasta ahora), aun asumiendo una posible separación entre propensión y sensibilidad al asco (en relación con esta separación, véase también Sandín, Chorot, Olmedo y Valiente, 2008a). En términos genéricos, por tanto, la sensibilidad al asco se referiría al grado en que un individuo siente asco en respuesta a diversos estímulos; en este sentido, la sensibilidad al asco es considerada como una variable de predisposición individual (i.e., las personas con elevada sensibilidad al asco poseen una tendencia mayor que otras personas a experimentar reacciones de asco o repugnancia ante las diversas situaciones relevantes). Actualmente se asume que la sensibilidad al asco constituye una tendencia con estabilidad temporal, que correlaciona moderada y positivamente entre padres e hijos ( $\mathrm{Da}$ vey, Foster y Mayhew, 1993).

\section{MANIFESTACIONES O COMPONENTES DEL ASCO}

De modo similar a lo que se ha indicado para las respuestas de miedo y ansiedad, el asco, como emoción básica, posee sistemas o componentes distintivos, entre los cuales al menos podrían diferenciarse los siguientes: (1) el fenomenológico, (2) el fisiológico o somático, (3) el conductual, y (4) el cognitivo. El componente fenomenológico comprende la experiencia personal, o percepción personal de la emoción de asco (p.ej., experiencia de náusea 
o repugnancia); constituye el elemento central de la emoción de asco, ya que sin él cualquiera de los restantes componentes perdería significado desde el punto de vista psicopatológico. Generalmente suele evaluarse mediante procedimientos de autoinforme (véase más adelante). La experiencia subjetiva de asco o repugnancia implica a los restantes componentes, esto es, síntomas somáticos (p.ej., síntomas de náuseas o vómito), síntomas cognitivos (p.ej., preocupación o evaluación negativa de los síntomas somáticos del asco), y síntomas comportamentales (p.ej., expresión facial o evitación). La experiencia de asco, al igual que la experiencia de miedo, suele asociarse a cierto grado de malestar emocional (experiencia emocional negativa).

El componente somático o fisiológico del asco se ha diferenciado del componente somatofisiológico del miedo. En contraste con el miedo, se ha sugerido que el asco se asocia a una activación del sistema nervioso parasimpático, lo cual se ha evidenciado a través de reducciones de la tasa cardiaca, de la presión sanguínea, de la tasa respiratoria y de la temperatura de la piel. También se ha constatado que durante las respuestas de asco se incrementa la salivación y la movilidad gastrointestinal, ambas precursoras de las náuseas y el vómito. (Olatunji y Sawchuk, 2005; Woody y Teachman, 2000).

Actualmente se investiga la posibilidad de que la emoción de asco se asocie a mecanismos neurofisiológicos específicos, independientes de los que ocurren en otras emociones. En este sentido, se ha sugerido que el córtex insular (ínsula anterior) podría constituir una estructura cerebral relacionada específicamente con el procesamiento de las emociones de asco (p.ej., procesamiento de las expresiones faciales de asco) (p.ej., Calder, Lawrence y Young, 2001; Phillips et al., 1997). Lo cual contrastaría y diferiría del procesamiento de la emoción de miedo, localizado en la amígdala. La cuestión, sin embargo, no parece que sea ni tan clara ni tan simple. Por ejemplo, la sensibilidad al asco (como variable de personalidad, evaluada mediante cuestionario de autoinforme) parece que influye sobre las respuestas cerebrales que dan los individuos a los estímulos repugnantes. A través de estudios basados en neuroimagen (fMRI - functional magnetic resonance imaging), se ha constatado que no es factible defender la dicotomía miedo vs. asco en paralelo con la dicotomía amígdala vs. ínsula, ya que ambas emociones parecen relacionarse con estructuras cerebrales comunes (y posiblemente comunes también a otras emociones). Diversos trabajos basados en neuroimagen han constatado una correlación positiva entre el nivel de sensibilidad al asco y la activación de ciertas estructuras cerebrales, tales como la amígdala (una estructura que parece relacionarse tanto con el miedo como con el asco) (véase Vaitl, Schienle y Stark, 2005).

Así mismo, cuando se tiene en cuenta el nivel de asco o miedo experimentado por los sujetos (mediante autoinforme), el procesamiento cerebral de los estímulos inductores de asco o miedo parece que implica a regiones cerebrales similares y distintas a la vez. Ambos tipos de estímulos emocionales generan activación del córtex occipital, del córtex prefrontal y de la amígdala. Sin embargo, aunque tanto los niveles subjetivos de miedo como de asco correlacionaban positivamente con la activación de estas tres áreas cerebrales, la activación de la ínsula sólo correlacionó de forma significativa con el nivel subjetivo de asco (autoinforme), lo cual sugiere un posible papel específico de esta estructura cerebral en el procesamiento de la emoción de asco (Stark et al., 2007). Llama la atención en este estudio que, aunque la amígdala era activada por ambos tipos de estímulos, la activación era mayor con los estímulos de asco que con los estímulos inductores de miedo. Estos y otros resultados de este grupo sugieren que existen ciertos solapamientos en el procesamiento cerebral del asco y del miedo en lo que concierne a determinadas regiones cerebrales (i.e., amígdala, córtex occipital y córtex prefrontal). Sin embargo, el hecho de que la activación de la amígdala sea mayor para el procesamiento del asco que del miedo supondría modificar la creencia general que asigna a la amígdala un papel preponderante y específico de procesamiento del miedo. Por otra parte, dado que el córtex insular parece relacionarse de forma específica con el procesamiento de las escenas inductoras de asco, los sistemas cerebrales que procesan el miedo y el asco son también (al menos parcialmente) disociables. 
El componente comportamental del asco presenta una característica propia, i.e., la expresión facial típica, y una característica común al miedo, i.e., la respuesta de evitación o escape. Tanto la expresión facial como las respuestas de evitación denotan el aspecto básico del asco, esto es, su carácter de rechazo del estímulo relevante (inicialmente el asco fue definido como el rechazo de la comida debido a la naturaleza u origen de ésta; Rozin y Fallon, 1987). Ambos fenómenos (expresión facial y evitación) reflejan el valor funcional adaptativo de las respuestas de asco, protegiendo al individuo del contacto no deseado o de la incorporación inapropiada de sustancias potencialmente peligrosas o en mal estado.

La expresión facial típica de la experiencia de asco o repugnancia es única y puede identificarse fácilmente a través de cualquier cultura, lo que sugiere su valor filogenético adaptativo. Tal expresión favorece el rechazo de sustancias que puedan ser incorporadas por las vías gustativa y olfativa, o la expulsión inmediata de sustancias nocivas que acaban de ser incorporadas (Rozin et al., 2000).

La principal tendencia a la acción que motiva la experiencia de asco es la evitación. En este sentido, parece haber cierto paralelismo entre el miedo y el asco. Ambas emociones suelen motivar la evitación o retirada, la primera (miedo) para reducir la sensación de peligro general, y la segunda (asco) para repeler cierta incorporación sensorial (olor, sabor, vista, sonido o tacto del estímulo). No obstante, ambas formas de evitación difieren en su finalidad; mientras que la evitación motivada por el miedo protege a la persona del peligro percibido, la evitación motivada por el asco se asocia con mayor frecuencia a la sensación o imaginación (Woody y Teachman, 2000). Estos autores asumen, sin embargo, que las dos formas de evitación pueden solaparse. Por ejemplo, la evitación motivada por el miedo en respuesta a las sensaciones es evidente en las reacciones a estímulos dolorosos, mientras que la evitación motivada por el asco en respuesta a peligro percibido es evidente en las reacciones a estímulos contaminantes.

Finalmente, el componente cognitivo del asco se ha investigado en menor grado que los componentes subjetivo (fenomenológico), fisiológico y conductual (Teachman, 2006). Como punto de partida para una posible diferenciación entre el miedo y el asco, Woody y Teachman (2000) sugirieron que la evaluación cognitiva de un estímulo como un peligro o una amenaza potenciales suele asociarse a una respuesta de miedo. Aunque las evaluaciones asociadas al asco pueden solaparse con el miedo (por compartir componentes de peligro), las evaluaciones de asco suelen focalizarse más específicamente en amenazas de contaminación (física o simbólica/social) que en rangos amplios de amenazas percibidas. En este sentido, se ha sugerido que las creencias de contaminación pueden mantenerse de forma rígida en el individuo, incluso aunque éste disponga de evidencia objetiva de lo contrario, lo cual ha sido etiquetado en forma de dos leyes mágicas («laws of sympathetic magic»), la ley del contagio y la ley de similitud (Rozin y Fallon, 1987; Rozin y Nemeroff, 1990). La ley del contagio indica que las cosas transfieren algunas de sus propiedades cuando tocan otras cosas, de modo que el efecto debido al contacto permanece aunque se haya eliminado la posible conexión («una vez en contacto siempre en contacto»). Por ejemplo, un objeto ensuciado por otro objeto se percibe siempre como sucio aunque posteriormente haya sido incluso esterilizado. La ley de similitud sugiere que las cosas que se asemejan a otra comparten propiedades fundamentales («contagio mágico»). De acuerdo con esta ley, los objetos objetivamente sanos son rechazados si recuerdan a otros objetos relevantes al asco. Por ejemplo, un dulce con forma de excremento de perro suele ser rechazado sistemáticamente.

La implicación de la evaluación cognitiva en la determinación las cosas que nos gustan y disgustan (o nos repugnan) denota, así mismo, que en las respuestas de asco el condicionamiento evaluativo (véase Levey y Martin, 1987; Merckelbach, de Jong, Arntz y Schouten, 1993) podría desempeñar un papel preponderante, en contraste con el condicionamiento pavloviano que está más implicado en las respuestas de miedo. Aunque la topografía de las respuestas de asco parecen ser universales, y aunque algunas de estas respuestas pueden darse de forma cuasi automática ante estímulos relevantes al asco preparados filogenéticamente, no cabe duda que existen grandes diferencias inter e intra culturales 
sobre lo que es asqueroso y sobre lo que no lo es, así como sobre las reacciones de los individuos a los estímulos desencadenantes de respuestas de asco.

\section{EVALUACIÓN Y DIMENSIONES RELEVANTES}

Como indicamos atrás, no todas las personas tienen la misma tendencia a experimentar reacciones de asco ante las mismas situaciones potencialmente desencadenantes. Por ello, la evaluación de las diferencias individuales en la predisposición al asco ha constituido un aspecto central de la investigación focalizada en el asco. Tal aspecto ha sido entendido como sensibilidad al asco; es decir, los individuos con mayor predisposición al asco serían los más sensibles a experimentar reacciones de asco. La elaboración de instrumentos apropiados para evaluar este constructo constituye, por tanto, un aspecto central en el momento actual de la investigación sobre el asco.

Un problema potencial, y una posible razón, de las controversias e inconsistencias que se han observado en algunos de los estudios llevados a cabo sobre la sensibilidad al asco y los síntomas y/o trastornos psicopatológicos podría consistir en la inadecuación de los instrumentos de evaluación disponibles. Rozin, Fallon y Mandell (1984) desarrollaron inicialmente una prueba de autoinforme de 24 elementos, el Disgust and Contamination Sensitivy Questionaire, primer instrumento para la evaluación de las experiencias de asco en el campo de la psicopatología. Puesto que esta escala únicamente se centraba en la evaluación de aspectos asociados a la comida, ha resultado de escasa utilidad para la evaluación de la sensibilidad al asco.

Haidt et al. (1994) elaboraron la Disgust Scale (DS) con la finalidad de proporcionar un instrumento de autoinforme más comprehensivo. Consta de 32 elementos que posibilitan evaluar las 8 dimensiones o áreas de sensibilidad al asco siguientes: (1) comida (p.ej., tomar comida en mal estado), (2) animales (p.ej., animales que viven en condiciones de suciedad, tales como las arañas o los gusanos), (3) productos corpora- les (p.ej., orina, saliva), (4) sexo (p.ej., incesto $\mathrm{u}$ otras conductas sexuales desviadas culturalmente), (5) trasgresión de la envoltura corporal (p.ej., ver los intestinos de una persona o cuerpos mutilados), (6) muerte (o cuerpos muertos), (7) contaminación mágica (contaminación improbable; p.ej., un trozo de chocolate con aspecto de excremento de perro), y (8) higiene (p.ej., tocar el asiento de la taza del water). Nuestro grupo ha traducido y validado este cuestionario a través de diversos trabajos (Sandín, Valiente y Chorot, 1999; Valiente, Sandín, Chorot, y Santed, 2004). Cada una de estas dimensiones se evalúa a través de 4 items, dos de los cuales son estimados dicotómicamente y dos según una escala tipo Likert.

Aunque la DS es la escala de sensibilidad al asco que hasta este momento más se ha empleado, no ha estado exenta de importantes críticas. Aparte de que la escala no parece cubrir adecuadamente las distintas facetas relacionadas con las experiencias de asco (Olatunji, Cisler, Deacon, Connolly y Lohr, 2007a; Olatunji et al., 2007e) y de la limitada fiabilidad de las subescalas, dos de sus principales críticas han tenido que ver con su estructura formal [los primeros 16 items se contestan según un formato de verdadero-falso, mientras que los 16 restantes se responden según una escala de repugnancia de 3 puntos $(0 / 1 / 2)$ ] y con sus dimensiones. No han sido replicados los 8 factores sugeridos inicialmente por Haidt et al. (1994) (no hay acuerdo sobre el número de sus dimensiones). Rozin et al. (2000) propusieron como alternativa una estructura de dos factores, que denominaron «asco esencial» (core disgust) y «recuerdo animal» (animal reminder disgust). El asco esencial se refiere a amenazas relacionadas con la contaminación, mientras que el asco de la dimensión de recuerdo animal reflejaría la aversión hacia los estímulos que sirven como recordatorios del origen animal de los humanos (p.ej., «Me molestaría tremendamente tocar un cuerpo muerto»).

Partiendo de las inconsistencias observadas en la DS, Olatunji et al. (2007e) llevaron a cabo recientemente una profunda revisión de la escala (Disgust Scale-Revised, DS-R), mediante la cual se han eliminado algunos items problemáticos de la DS y se ha uniformado el formato 
de respuesta. De este modo, la DS-R se ha reducido a 25 items. Los análisis finales efectuados sugieren tres dimensiones de sensibilidad al asco: (1) asco esencial, (2) recuerdo animal, y (3) contaminación interpersonal, las cuales han sido validados recientemente (Olatunji, Haidt, McKay y David, 2008). Esta nueva versión revisada (DS-R) ha sido adaptada al español recientemente por nuestro grupo (Sandín, Valiente y Chorot, 2008b; véase también la dirección web de la escala: http://people.virginia.edu/ jdh6n/disgustscale.ht$\mathrm{ml})$. La dimensión de asco esencial incluye la repugnancia a alimentos, animales y productos corporales (p.ej., «Si veo a alguien vomitar se me pone mal el estómago»). La dimensión de recuerdo animal incluye la repugnancia asociada a la muerte y a violaciones de la envoltura corporal («Me molestaría tremendamente tocar un cuerpo muerto»). La dimensión de contaminación interpersonal se refiere al asco ante la posible transmisión interpersonal de productos contaminantes («Evitaría ir a mi restaurante favorito si descubriera que el cocinero tiene catarro»). Los resultados de los estudios factoriales sobre la DS-R no apoyan consistentemente la estructura de tres factores sugerida por Olatunji et al. (2007e), habiéndose sugerido entre uno (van Overveld, 2008) y tres o cuatro factores (Valiente, Sandín, Olmedo, Chorot y Santed, 2008a; Valiente et al., 2008b). Los datos sobre fiabilidad y validez con la versión española sugieren aceptables niveles de fiabilidad (Valiente et al., 2008a,b) y de validez convergente y discriminante a través de correlaciones con otros constructos como la afectividad positiva y negativa, los tipos de miedos, y los síntomas obsesivo-compulsivos (Valiente et al., 2008a).

La Disgust Emotion Scale (DES; Kleinknecht, Kleinknecht, y Thorndike, 1997) fue presentada como una alternativa a la DS. A partir de 30 items permite evaluar cinco áreas o dimensiones de contenido del asco (comida, pequeños animales, inyecciones/sangre, mutilaciones y muerte, y olores). Cada una de estas subescalas está constituida por 6 items, los cuales son estimadas por el sujeto según una escala tipo Likert de 5 puntos (0-4). Aunque se han indicado que la DES posee buenos niveles de fiabilidad (consistencia interna), se ha señalado que ésta aparece sobreestimada por la existencia de items redundantes (correspondientes fundamentalmente a las dimensiones de inyecciones/sangre y mutilación/muerte) (Olatunji, Sawchuk, de Jong y Lohr, 2007c). No obstante, tal vez el principal problema de la DES sea su elevado nivel de confusión de medidas: no pocos de sus items se confunden con síntomas de ansiedad o miedo. Si a esto añadimos que, aun siendo contextualmente dependiente (la DES únicamente evalúa reacciones de asco a estímulos o situaciones específicos), la escala evalúa un bajo abanico de dominios o áreas del asco, podríamos decir que su utilidad para la investigación y la aplicación clínica es limitada.

En general, aunque en distinto grado, todas las escalas comentadas arriba evalúan el asco asociado a desencadenantes específicos. Este hecho tiene el problema de que pueden solaparse la evaluación de la sensibilidad al asco y la evaluación de síntomas psicopatológicos (p.ej., a través de cuestionarios que miden síntomas de ansiedad), debido a que en ambos casos los items se refieren a objetos similares (la sangre, heridas, inyecciones, animales, etc.) (fenómeno conocido como confusión de medidas). Como alternativa a estos instrumentos, Cavanagh y Davey (2000) desarrollaron una nueva escala de autoinforme que denominaron Disgust Propensity and Sensitivity Scale (DPSS). Esta escala fue concebida como una escala de propensión al asco libre contextualmente, con objeto de evitar dicha confusión. Por otra parte, los autores incluyeron la evaluación negativa que suele hacer el individuo sobre las experiencias de asco. De este modo, los autores separan entre la tendencia a experimentar asco (propensión al asco) y el desagrado o preocupación ante tal experiencia (sensibilidad al asco). La idea, aunque importante, no es nueva, pues es similar a la distinción que se ha hecho entre propensión a la ansiedad (rasgo de ansiedad) y sensibilidad a la ansiedad (Chorot et al., 1997; Sandín, Chorot y McNally, 2001; Sandín, Chorot, Santed y Valiente, 2002; Sandín, Chorot, Valiente, Santed y Lostao, 2004; Sandín, Valiente, Chorot y Santed, 2005, 2007a,b; Taylor, 1999).

Tal distinción supone una ruptura con lo que comúnmente ha venido suponiendo una identificación de la sensibilidad al asco con la pre- 
disposición o vulnerabilidad al asco. Partiendo de este presupuesto, la escala pretende evaluar estas dos características, es decir, hasta qué punto el individuo siente repugnancia con facilidad ante cualquier estímulo (propensión), y en qué medida experimenta como desagradable o molesta la experiencia de sentir el asco (sensibilidad). Esta diferenciación es interesante, aunque apenas ha sido investigada todavía (aparte del peligro de poder confundirse la sensibilidad al asco con la sensibilidad a la ansiedad). Al margen de este posible problema, tal separación es conceptual y operativamente asumible científicamente. Recientemente se ha publicado una forma depurada de la escala, la Disgust Propensity and Sensitivity Scale-Revised (DPSS$\mathrm{R}$ ), pasando de 23 items (forma inicial experimental; Cavanagh y Davey, 2000) a los 16 items de esta nueva versión revisada por van Overveld et al., 2006). Cada subescala (propensión al asco y sensibilidad al asco) consta de 8 items, los cuales son estimados por el sujeto según una escala Likert de 1-5.

Existen datos que apoyan la estructura factorial (dos factores separados de propensión y sensibilidad, respectivamente), la fiabilidad y la validez convergente y discriminante de las versiones holandesa (véase van Overveld, 2008), inglesa (Olatunji et al., 2007a) y española (Sandín et al., 2008a) de la DPSS-R. Nuestros análisis factoriales exploratorios y confirmatorios apoyan la hipótesis de que las diferencias individuales en la emoción del asco pueden entenderse desde dos planos diferentes, aunque relacionados, denominados propensión (o predisposición) y sensibilidad. Según esto, una persona puede ser muy propensa a experimentar reacciones de asco, y sin embargo no ser sensible (o ser poco sensible) a los síntomas del asco. Nuestros datos proporcionan también evidencia empírica suficientemente relevante en apoyo de la validez predictiva, ya que tanto la propensión como la sensibilidad contribuían de forma única en la predicción de los miedos y los síntomas del trastorno obsesivo-compulsivo (véase Sandín et al., 2008a, para una exposición más amplia y detallada). Así mismo, cabe resaltar que uno de nuestros resultados sobre la validación factorial de la DPSS-R fue la depuración de ésta y la propuesta de una forma re- visada de la misma que hemos denominado DPSS-R-12. Las correlaciones entre ambas formas (i.e., entre subescalas similares) son elevadas, lo que indica que pueden emplearse de forma intercambiable. Sin embargo, la valoración de los diferentes datos presentados en este trabajo sobre esta forma abreviada sugiere que en muchos aspectos es superior psicométricamente a la versión completa. Por ejemplo, su estructura factorial es más coherente y robusta, y el solapamiento entre sus dos subescalas es menor. Si a esto añadimos que la forma abreviada posee un $25 \%$ menos de items, parece razonable y justificado sugerir que la DPSS-R-12 podría ser la escala de elección tanto para la investigación como para la aplicación clínica (en ambos ámbitos es necesario disponer de instrumentos válidos y fiables, pero de rápida aplicación) (Sandín et al., 2008a).

Partiendo de que la emoción de asco no parece ser homogénea y retomando la idea sobre las posibles dimensiones o dominios del asco, si bien no existe actualmente un claro acuerdo sobre su número y/o identificación, parece existir cierto consenso sobre el mínimo de dimensiones relevantes. En este sentido, diversos autores han señalado la conveniencia de asumir al menos tres tipos de áreas o dimensiones separadas relacionadas con la emoción del asco, las cuales han sido denominadas como: asco esencial (core disgust), recuerdo animal (animal-reminder disgust) y socio/moral (Simpson, Carter, Anthony y Overton, 2006; Olatunji y Sawchuk, 2005; Olatunji et al., 2008; van Overveld, 2008). Olatunji et al. (2007e, 2008) sugieren la existencia de una dimensión adicional que denominan asco interpersonal, o contaminación interpersonal (véanse atrás nuestros comentarios sobre la DS-R), si bien los datos son menos consistentes para esta dimensión. El denominado asco socio/moral se produce cuando se violan las normas morales o sociales (violaciones de la autonomía y/o dignidad de otras personas) (p.ej., asco asociado al racismo, hipocresía, deslealtad, incesto, abuso sexual, etc.). En contraste con las restantes formas de asco, el asco sociomoral es más dependiente de las normas culturales y suele asociarse a emociones de ira más que a emociones de ansiedad o miedo (Simpson et al., 2006). En suma, las diferencias indivi- 
duales en la emoción del asco pueden entenderse tanto desde dimensiones establecidas contextualmente (tres o cuatro dimensiones básicas) como desde dimensiones más generales no vinculadas a estímulos u objetos específicos (dimensiones de propensión y sensibilidad).

\section{Sensibilidad al asco y psicopatología: Síntomas y trastornos de ansiedad}

Si el asco actúa para prevenir la contaminación y la enfermedad (Rozin y Fallon, 1987), cabe pensar que éste podría jugar un papel importante en aquellos trastornos de ansiedad, $\mathrm{u}$ otros trastornos psicopatológicos, que implican componentes de contaminación y enfermedad, o en relación con los estímulos asociados a una posible contaminación o enfermedad. Debe señalarse, sin embargo, que el estudio de la emoción del asco desde el punto de vista psicopatológico ha recibido mucha menos atención que otras emociones negativas básicas, tales como el miedo (o ansiedad), la tristeza (o depresión) y la ira (o agresión). El célebre artículo de Phillips, Senior, Fahy y Davey (1998) «Disgust: The forgotten emotion in psychiatry» es sólo un signo de la actual preocupación sobre esta cuestión. Así, desde la publicación de este artículo hasta el posterior artículo de McNally (2002) «Disgust has arrived» sólo pasaron cuatro años, lo cual denota el creciente interés por la investigación sobre el asco y su relación con la psicopatología. Aunque, como señalaron Phi1lips et al. (1998), el asco parece relacionarse con la mayor parte de los trastornos psicológicos, recientemente se ha desarrollado un cuerpo de evidencia relevante que sugiere una estrecha relación entre la sensibilidad al asco y diversos trastornos de ansiedad, particularmente ciertas fobias y miedos específicos y determinados tipos del trastorno obsesivo-compulsivo.

\section{Sensibilidad al asco y miedos y fobias a los animales}

El interés por la investigación sobre las relaciones entre el asco y los trastornos de an- siedad parte básicamente de los estudios del grupo de Davey (p.ej., Davey, 1992, 1994; Davey et al., 1993; Matchett y Davey, 1991; Ware, Jain, Burgess y Davey, 1994). Partiendo de la observación de que los individuos con fobias a ciertos animales, aparte de sus reacciones típicas de miedo, suelen experimentar también reacciones de asco, Matchett y Davey (1991) propusieron que la conducta de evitación de dichos individuos no es motivada únicamente por el miedo sino también por el asco. Estos autores evaluaron la sensibilidad al asco y la contaminación y encontraron que correlacionaban de forma elevada con los niveles de miedo a ciertos tipos de animales, en concreto a los animales «relevantes al miedo» pero no peligrosos, tales como las arañas, las ratas, y las cucarachas. En cambio, la sensibilidad al asco no correlacionaba con el miedo a los animales depredadores, tales como el tigre, el león, el oso o el tiburón. Posteriormente Davey (1992) demostró que el asco se asociaba de modo similar a dichos animales relevantes al miedo (no peligrosos) y a los animales que suelen provocar el asco de modo natural (p.ej., babosas, caracoles, orugas, gusanos, ranas, etc.). Con objeto de examinar la posible relación causal entre la sensibilidad al asco y el miedo a los animales, Webb y Davey (1992) evaluaron experimentalmente el nivel de miedo asociado a escenas «asquerosas» (video con escenas repugnantes), y demostraron que la inducción de asco producía un incremento del miedo sólo a los animales considerados normalmente como relevantes al miedo pero no peligrosos (rata, araña, culebra) o relevantes al asco (babosa, caracol, gusano), no a los animales relevantes al peligro (león, tigre, tiburón); lo cual sugiere una relación causal entre el asco y el miedo. Finalmente, otra de las importantes aportaciones del grupo de Davey fue demostrar que existía una transmisión familiar de la sensibilidad al asco, caracterizada por una notable semejanza en la sensibilidad al asco entre padres e hijos, en la que participaban tanto elementos genéticos como mecanismos de aprendizaje (Davey et al., 1993). En este estudio, Davey et al. no sólo constaron correlaciones significativas en las puntuaciones de asco entre padres e hijos, sino que también 
demostraron que la sensibilidad al asco de los padres predecía el nivel general de miedo en los hijos, y especialmente el miedo a las arañas.

Tras estas series de estudios en los que se demostraba una estrecha relación (incluida la relación etiológica) entre la sensibilidad al asco y el miedo/fobia a ciertos animales, los autores concluyeron que la sensibilidad al asco favorece la adquisición de miedos a determinados animales asociados a la transmisión de suciedad, enfermedad e infección, sugiriendo la denominada «hipótesis de evitación de la enfermedad» (Davey, 1992, 1994; Ware et al., 1994), hipótesis que, aunque ha sido sugerida como alternativa a la teoría de la preparación de las fobias (evitación de animales peligrosos - animales depredadores), en realidad representa más bien una extensión de ésta (véase Davey, 1992; Sandín, 1997; Sandín y Chorot, 1999; Sandín, Chorot, Valiente y Santed, 1998; Valiente, Sandín y Chorot, 2003; Valiente, Sandín, Chorot y Tabar, 2003).

Las investigaciones sobre la estructura de los miedos a los animales han indicado la presencia de al menos dos factores de orden inferior: (1) miedos a los animales de tipo depredador (lobos, osos, etc.), y (2) miedos a pequeños animales no depredadores pero repugnantes (ratas, arañas, serpientes, etc.) (Valiente, 2001; Valiente et al., 2003; Ware et al., 1994). En un estudio transcultural que incluía a siete países (Reino Unido, India, USA, Holanda, Corea, Hong Kong, y Japón; Davey et al., 1998), dirigido a estudiar las dimensiones de los miedos, se constató que éstos se agrupaban de forma consistente en los tres factores siguientes (Sandín, 1999): (1) Miedos a animales inofensivos (animales irrelevantes al miedo). Constituía el primer factor, e incluía animales más o menos comunes/domésticos y poco relevantes al miedo, tales como el perro, el cerdo, la oveja, el gato, la vaca, etc. (2) Miedos a animales depredadores (animales fóbico-relevantes). Esta dimensión configuraba el segundo factor, y se refería a miedos a animales considerados habitualmente como peligrosos por su fiereza, como el león, el oso, el caimán, el cocodrilo, el tigre, el lobo, el tiburón, etc. Y (3) miedos a los animales repugnantes (animales relevantes al asco). La dimensión constituida por los miedos a animales asquerosos determinaba el tercero de los factores. Pertenecían a este factor los miedos a animales como la cucaracha, la araña, el escarabajo, el gusano, la lombriz, la lagartija, el murciélago, la rata, etc. La obtención de este tipo de factor a través de las seis culturas indicadas, como dimensión separada de otras categorías de miedos a los animales, es consistente con el concepto de «sensibilidad al asco» y con la hipótesis de que el asco es una emoción universal que puede asociarse de forma consistente a estímulos (animales) que podrían estar vinculados a la contaminación y transmisión de enfermedades, o que poseen características que recuerdan estímulos primarios de asco como la mucosidad (Davey, 1992; Matchett y Davey, 1991; Sandín, 1997, Sandín y Chorot, 1999).

La hipótesis de evitación de la enfermedad sugerida por Davey es consistente con el hecho de que muchos pequeños animales no son peligrosos en absoluto (McNally, 2002), y proporciona una nueva perspectiva psicopatológica sobre ciertas fobias basada en la emoción del asco. Aunque esta hipótesis puede aplicarse a varios tipos de fobias, la mayor parte de la investigación sobre sensibilidad al asco y fobias a los animales ha gravitado sobre la fobia a las arañas. Actualmente existe abundante evidencia que sugiere que el asco y la sensibilidad al asco covarían y/o están implicadas de forma consistente en la etiología y/o mantenimiento de la fobia a las arañas o del elevado miedo a las arañas (véase, p.ej., Olatunji y Sawchuk, 2005; Woody y Teachman, 2000; van Overveld, 2008). En este sentido, la sensibilidad al asco se ha encontrado sistemáticamente elevada en los pacientes con fobia a las arañas (p.ej., de Jong y Merckelbach, 1998; Merckelbach, de Jong, Arntz y Schouten, 1993; Mulkens, de Jong y Merckelbach, 1996; Sawchuk, Lohr, Tolin, Lee y Kleinknecht, 2000), incluso en niños y adolescentes con este tipo de fobia (de Jong, Andrea y Muris, 1997). Este tipo de evidencia sugiere que la sensibilidad al asco puede predisponer al miedo a las arañas.

En diversos estudios, con distintos paradigmas metodológicos, se ha evaluado la sensibilidad al asco y/o las respuestas de asco asociadas a las respuestas de miedo. Se ha demostrado que la sensibilidad al asco está estrechamente re- 
lacionada con la conducta de evitación a las arañas (Olatunji y Deacon, 2008), así como también que la tendencia a evitar las arañas es mejor predicha por el asco que por el miedo (Woody, McLean y Klassen, 2005). Cuando los pacientes con fobia a las arañas, a con elevado nivel de miedo a las arañas (análogos clínicos), son expuestos a estímulos relevantes (p.ej., imágenes de arañas), suelen experimentar tanto emociones de miedo como de asco (p.ej., Sawchuk, Lohr, Westendorf, Meunier y Tolin, 2002; Thorpe y Salkovskis, 1998; Vernon y Berenbaum, 2002). También se ha visto, mediante paradigmas de asociación implícita (implicit assocition tasks, IAT), que los individuos con miedo/fobia a las arañas suelen asociar las arañas a atributos de miedo y de asco (p.ej., Huigding y de Jong, 2007; van Overveld, de Jong y Peters, 2006), pudiendo ser estos modificados mediante terapia de conducta (la reducción del miedo es paralela a reducción del asco a las arañas; de Jong et al., 1997).

En conjunto, la investigación sobre los miedos y fobias específicas sugieren que existe una relación muy estrecha entre los miedos y/o fobias a ciertos tipos de animales (animales pequeños no peligrosos, incluidas especialmente las arañas) y la sensibilidad y reacciones de asco. Las fobias a estos animales no parece que se basen únicamente en respuestas de miedo, pues de hecho la conducta de evitación típica de estas fobias parece deberse a ambas emociones (asco y miedo). La experiencia de las respuestas fóbicas implica una combinación de asco y miedo. La sensibilidad al asco parece ser un factor de riesgo para el desarrollo y mantenimiento de las fobias a estos tipos de animales. La evaluación de la amenaza que llevan a cabo los individuos con fobias a las arañas parece incluir tanto atributos de miedo como de asco. Se han sugerido diversas vías a través de las cuales ciertos animales adquieren la propiedad de desencadenar asco, entre las que se incluyen las siguientes: (1) por asociarse directa o indirectamente con la transmisión de enfermedad e infección (p.ej., las ratas); (2) para algunos animales porque poseen características que recuerdan a los estímulos evocadores naturales de respuestas de asco, tales como las heces o la mucosidad (p.ej., las ba- bosas, los gusanos); (3) la tercera categoría se refiere a aquellos animales que, bien se asocian contingentemente a la suciedad, la enfermedad o el contagio, o bien actúan como señales de suciedad, enfermedad o infección (a esta categoría podrían corresponder las arañas, animales que, desde la Edad Media, han sido asociados a la muerte, la enfermedad y las plagas) (Ware et al., 1994).

\section{Sensibilidad al asco y miedos y fobias a la sangre-inyecciones daño}

Como vimos en el epígrafe anterior, las reacciones fóbicas no siempre implican reacciones estrictas de miedo, pues a veces (en ciertas fobias a los animales) implican también, y de forma considerable, las reacciones de asco. Un ejemplo claro de fobia específica en la que están implicadas las reacciones de asco es la fobia del tipo sangre-inyecciones-daño (SID). Page (1994) sugirió que en las fobias SID el asco jugaba un papel incluso más relevante que en las fobias a los animales (véase Sandín, 1997). La exposición a la sangre, mutilaciones, heridas y otras situaciones similares suele desencadenar reacciones de náusea, aversión y asco, a veces incluso en ausencia completa de reacciones de miedo.

De modo similar a lo que se ha observado en los estudios sobre miedos y fobias a los animales, la evidencia actual sugiere una estrecha asociación entre el asco y las fobias SID. Esta asociación se ha demostrado a través de diversos tipos de evidencia, entre los que se incluye (1) la observación de elevados niveles de sensibilidad al asco en individuos con fobias SID (Olatunji, Sawchuk, Lohr, y de Jong, 2004; Page, 1994; Sawchuk et al., 2000; Schienle, Schäfer, Walter, Stark y Vaitl, 2005; van Overveld, 2008); (2) la generación de respuestas emocionales de asco ante la exposición a estímulos relevantes (estímulos SID), en individuos fóbicos o análogos clínicos SID (Page, 1994, 2003; Sawchuk et al., 2002; Schienle, Stark y Vaitl, 2001; Tolin, Lohr, Sawchuk y Lee, 1997; van Overveld, 2008); y (3) la asociación de expectativas de asco ante estímulos SID (de Jong y Peters, 2007; van Overveld, 2008). Un aspecto a resaltar so- 
bre la provocación de respuestas de asco ante la presencia de estímulos fóbicos relevantes es que, en individuos con fobia SID, la presentación de material SID suele provocar mayores respuestas de asco que de miedo; lo cual contrasta con las respuestas de los fóbicos a las arañas, los cuales suelen emitir respuestas similares de asco y de miedo ante la presentación de estímulos relevantes (i.e., arañas) (Sawchuk et al., 2002; Tolin et al., 1997). Esto sugiere que, mientras que en las fobias a los animales el asco y el miedo parecen estar igualmente implicados, en las fobias SID el asco podría desempeñar un papel más importante que el miedo, jugando aquél un papel destacado como factor de riesgo para el desarrollo y/o mantenimiento de este tipo de fobias.

Una característica de las fobias SID, que no suele darse en otros tipos de fobia, es su asociación con el desmayo o síncope vasovagal (la mayoría de los individuos con fobia SID suele experimentar episodios parciales o totales de desmayo). El síncope vasovagal incluye una segunda fase en la se produce un incremento de la actividad del sistema nervioso parasimpático (activación del vago; la primera fase se asocia a un incremento del simpático) que suele llevar a la ocurrencia del desmayo (asociado a una fuerte caída de la presión sanguínea). Las similitudes entre las respuestas fisiológicas características que se dan en las fobias SID y las respuestas asociadas a las reacciones de asco (en ambos casos se dan las dos fases indicadas) constituyen la base del modelo de Page (1994, 2003) sobre la conexión entre el asco, el desmayo y las fobias SID (véase Sandín, 1997). Desde este punto de vista, este autor ha propuesto que el asco, a través de las respuestas parasimpáticas, actuaría como mediador de las respuestas fóbicas SID.

\section{Sensibilidad al asco y trastorno obsesivo-compulsivo}

El trastorno obsesivo-compulsivo (TOC) implica diversos tipos de síntomas, entre los que se incluyen los relacionados con la limpieza y la contaminación (obsesiones de limpieza y compulsiones de lavado) (Belloch, Morillo y García-
Soriano, 2006). Los pensamientos intrusos recurrentes de contaminación suelen afectar al $50 \%$ de los pacientes diagnosticados de TOC, y son especialmente frecuentes entre los pacientes con rituales compulsivos de limpieza y lavado (Olatunji y Sawchuk, 2005; Olatunji et al., 2004). Un aspecto importante en relación con esta cuestión es que bastantes pacientes diagnosticados de TOC, entre los que predominan las compulsiones de lavado, no suelen exhibir sentimientos de miedo sino más bien sentimientos de asco referidos a los objetos «contaminantes» (suelen etiquetar a estos objetos como asquerosos, más que como objetos temidos) (Tallis, 1996). Esta conexión que parece darse entre miedo a la contaminación, reacciones de asco y TOC podría interpretarse desde el modelo de evitación de la enfermedad planteado originalmente por Davey (Davey, 1992, 1994; Ware et al., 1994) para explicar ciertas fobias específicas (véase atrás). De hecho, estos autores (Ware et al., 1994) ya habían constatado que el miedo a los animales relevantes al asco, aunque no el miedo a los animales depredadores, correlacionaba con las compulsiones de limpieza, pero no con otros síntomas del TOC no relacionados con la contaminación. Si, como indica la teoría de evitación de la enfermedad, las respuestas de asco constituyen un mecanismo para prevenir la transmisión de enfermedad, cabe suponer que existan niveles elevados de sensibilidad al asco asociados tanto a las obsesiones de contaminación como a las compulsiones de limpieza.

Diversos estudios han observado correlaciones positivas y moderadas entre el nivel de sensibilidad al asco y la sintomatología obsesivocompulsiva (véase Berle y Phillips, 2006). Tales correlaciones se han documentado a partir de muestras de población no clínica (p.ej., Olatunji et al., 2004; Tolin, Woods y Abramowitz, 2006; Thorpe, Patel y Simonds, 2003) y con pacientes diagnosticados de TOC (Woody y Tolin, 2002), así como también a partir de muestras de niños y adolescentes (Muris et al., 2000; Muris, Merckelbach, Schmidt y Tierney, 1999; Muris, van der Heiden y Rassin, 2008); también se han encontrado correlaciones significativas entre sensibilidad al asco y contaminación mental (Herba y Rachman, 2007). No obstante, el tamaño de estas correlaciones suele reducirse sig- 
nificativamente cuando se controla el efecto del rasgo de ansiedad, permaneciendo relevantes fundamentalmente las correlaciones para los síntomas del TOC relacionados con el miedo a la contaminación (preocupaciones de contaminación ) y las compulsiones de limpieza (p.ej., Tolin et al., 2006). Las obsesiones de contaminación y las compulsiones de limpieza se relacionan de forma particularmente intensa con la sensibilidad al asco (Berle y Phillips, 2006), lo cual es consistente con el modelo de evitación de la enfermedad.

Tanto los pacientes con diagnóstico de TOC (Woody y Tolin, 2002), como los individuos con elevado nivel de miedo a la contaminación (análogos clínicos; Deacon y Olatunji, 2007; Olatunji, Lohr, Sawchuk y Tolin, 2007b), tienden a exhibir superiores niveles de sensibilidad al asco que los individuos normales. Más aun, la sensibilidad al asco ha resultado ser un buen predictor de las conductas de lavado compulsivo y chequeo (Manzini, Gragnani y D'Olimpio, 2001; Olatunji et al., 2004; Thorpe et al., 2003), así como también del miedo a la contaminación y la conducta de evitación en individuos sometidos a pruebas de evitación conductual tras la presentación de material inductor de asco (Deacon y Olatunji, 2007). Varios estudios han sugerido que la sensibilidad al asco puede actuar como mediador entre las preocupaciones de contaminación y la conducta de evitación (Deacon y Olatunji, 2007; Olatunji et al., 2007b). Así pues, el asco parece poseer un valor funcional en la etiología y mantenimiento de las obsesiones y compulsiones relacionadas con la contaminación, a partir de su papel mediacional entre la percepción de contaminación y las respuestas de evitación (p.ej., compulsiones de limpieza), y a través de un incremento de la percepción de amenaza (preocupación o miedo a la contaminación). Las dos leyes mágicas propuestas por Rozin (ley de similitud y ley de contacto; Rozin y Fallon, 1987; Rozin y Nemeroff, 1990), descritas atrás, podrían estar particularmente implicadas en la percepción de la amenaza de contaminación en los individuos con TOC. Aparte del estudio de Tolin, Worhunsky y Maltby (2004) centrado en la ley de contagio, no conocemos otros trabajos que hayan investigado directamente estas leyes asociadas al TOC.

\section{ALGUNOS PUNTOS DE INTERÉS QUE EXIGEN ULTERIORES INVESTIGACIONES}

Hasta aquí hemos visto que el asco y la sensibilidad al asco se relacionan de forma muy estrecha con determinados síntomas y trastornos de ansiedad, en concreto con ciertos tipos de miedos y fobias y con los síntomas y tipos del TOC en los que tienen una papel preponderante las manifestaciones relacionadas con el miedo a la contaminación (obsesiones de contaminación y compulsiones de lavado), pudiendo desempeñar un papel relevante en la etiología y mantenimiento de estos trastornos. Los estudios llevados a cabo sobre la sensibilidad al asco desde el marco de la psicología clínica y de la salud reflejan algunas cuestiones importantes que necesariamente exigen una mayor atención, y que sin duda justifican posteriores estudios. Vamos a referirnos brevemente a estos aspectos.

\section{Sobre las dimensiones de sensibilidad al asco}

Una primera cuestión que justifica mayor atención es la investigación sobre las dimensiones de sensibilidad al asco. La heterogeneidad de los estímulos desencadenantes de reacciones de asco sugiere que éste es un fenómeno multidimensional. Teóricamente se han propuesto las cuatro dimensiones siguientes: (1) asco esencial (core disgust), (2) recuerdo animal (animal-reminder disgust), (3) asco interpersonal y (4) asco sociomoral (Olatunji et al., 2008; Rozin et al., 2000; van Overveld, 2008). Los desencadenantes del asco esencial se caracterizan por una amenaza real o percibida respecto a una posible incorporación oral junto a una reacción de repugnancia. Comidas en mal estado, productos orgánicos de desecho (p.ej., heces) y pequeños animales, particularmente aquellos asociados a la basura y restos orgánicos (p.ej., ratas, cucarachas) corresponden a esta categoría. Esta forma de asco ha dado lugar a otras formas (tal vez por influencias culturales-evolutivas) de asco, tales como el asco de recuerdo animal, el asco interpersonal y el asco sociomoral. Los estímulos desencadenantes de asco 
de recuerdo animal son «recordatorios» de nuestra propia mortalidad inherente a nuestra naturaleza animal (p.ej., violaciones de la envoltura corporal, la muerte y prácticas relacionadas con el sexo). El asco interpersonal se asocia a situaciones en las que se produce contacto con individuos desconocidos, enfermos, o contaminados por la enfermedad o el infortunio, o ante la posibilidad de intimidad con ciertas personas. Finalmente, el asco sociomoral es una reacción a las clases sociales inferiores o a violaciones de la moral (p.ej., racismo, paidofilia, etc.; contribuye a la conservación de los valores sociales).

Aunque se han propuesto clasificaciones derivadas teóricamente, como indicamos atrás los estudios factoriales basados en las escalas de evaluación actuales no son totalmente consistentes a la hora de determinar las dimensiones o dominios básicos del asco o sensibilidad al asco. No obstante, la determinación precisa de estas dimensiones es un reto importante, ya que cada dimensión o faceta del asco podría relacionarse de forma diferente con los distintos problemas psicológicos o trastornos. En este sentido, se ha sugerido que las dos formas principales de sensibilidad al asco (esencial y recuerdo animal) parecen relacionarse de forma diferencial con la psicopatología. Por ejemplo, la fobia a las arañas se ha asociado con la predisposición al asco esencial, mientras que las fobias SID se ha relacionado más estrechamente con la propensión al asco de recuerdo animal (de Jong y Merckelbach, 1998). No obstante, los datos sobre la diferenciación entre las dimensiones de asco y los distintos síntomas y trastornos fóbicos aún no son concluyentes (véase van Overveld, 2008).

También se ha investigado la posibilidad de que las distintas formas de asco se asocien diferencialmente a los síntomas del TOC. A este respecto, aunque Olatunji et al. (2004) no encontraron diferencias en un estudio con participantes no clínicos (cualquier tipo de asco predecía por igual los síntomas de contaminación), Woody y Tolin (2002), partiendo de una muestra clínica, constataron que los pacientes TOC con compulsiones de limpieza puntuaban de forma más elevada que los controles sólo en las subescalas de sensibilidad al asco de animales, productos corporales, y contaminación mágica de la DS. Tolin et al. (2006) encontraron, en una muestra de participantes no clínicos, que los síntomas de compulsiones de lavado correlacionaban de forma significativa con todas las subescalas de la DS, excepto con trasgresión de la envoltura corporal; no obstante, las correlaciones más elevadas fueron con las subescalas de higiene y productos corporales. En un trabajo más reciente, Olatunji et al. (2007b) concluyeron que los análogos TOC exhibían elevados niveles de sensibilidad al asco en la mayoría de las subescalas de la DS, si bien las principales diferencias entre los análogos TOC y los que puntuaban bajo en síntomas TOC se dieron para las subescalas de productos corporales, higiene, $\mathrm{y}$ animales.

Se observa, por tanto, que aunque la investigación sobre la implicación diferencial de las facetas del asco es preliminar, ciertas dimensiones de la sensibilidad al asco parecen relacionarse de forma más específica que otras con los síntomas y trastornos psicopatológicos (miedos, fobias y TOC). Una dimensión de sensibilidad al asco no incluida en la DS, el asco sociomoral, parece diferir notablemente del asco esencial, ya que se ha asociado más estrechamente con reacciones de ira y depresión, en contraste con las reacciones de miedo (más estrechamente relacionadas con el asco esencial) (Simpson et al., 2006). Futuras investigaciones, no obstante, deberán clarificar mejor el alcance psicopatológico de las diferentes dimensiones del asco.

\section{Propensión versus sensibilidad}

Una segunda línea de desarrollo futuro concierne a la separación entre propensión y sensibilidad al asco. Aunque aún se sigue identificando la sensibilidad al asco con la predisposición (propensión) a experimentar reacciones de asco, recientemente van Overveld (2008; van Overveld et al., 2006; véase también Sandín et al., 2008a) ha sugerido una separación entre propensión y sensibilidad. Como indicamos atrás, la propensión al asco es definida como la tendencia individual general a experimentar la emoción de asco (con independencia de cuales sean las si- 
tuaciones evocadoras). La sensibilidad al asco, en contraste, se define como la tendencia del individuo a experimentar como desagradable la experiencia del asco. Los niveles elevados de sensibilidad al asco pueden motivar al individuo a evitar objetos o situaciones específicos debido al malestar producido por el asco experimentado. Aunque la información es aún preliminar, los trabajos llevados a cabo por van Overveld et al. (van Overveld, 2008) sugieren que la propensión y la sensibilidad podrían relacionarse diferencialmente con algunos problemas psicopatológicos. Específicamente hablando, y según estos autores, la propensión y sensibilidad tienen en común su relación positiva (así como también su capacidad predictiva) con los miedos SID y con los síntomas del TOC. Sin embargo, difieren en algunos aspectos: el miedo a las arañas es mejor predicho por la propensión, mientras que el miedo a vomitar y los síntomas de trastornos alimentarios se asocian más específicamente con la sensibilidad al asco (véase van Overveld, 2008).

En nuestro reciente trabajo con población no clínica sobre la sensibilidad al asco y los síntomas de ansiedad (Sandín et al., 2008a) encontramos que, tras controlar el efecto del afecto negativo y la sensibilidad a la ansiedad, tanto la propensión como la sensibilidad al asco correlacionaban de forma moderada y significativa con los miedos (interpersonales, SID, a los animales, y agorafóbicos) y síntomas globales del TOC. Cuando examinamos las correlaciones entre propensión y sensibilidad y los distintos tipos de síntomas TOC, la principal diferencia que observamos entre predisposición y sensibilidad fue que únicamente la sensibilidad correlacionaba de forma significativa con los pensamientos obsesivos de daño a sí mismo/otros, a la vez que las obsesiones de contaminación y las compulsiones de lavado se relacionaban de forma más intensa con la propensión que con la sensibilidad. Los análisis de regresión indicaron que (1) la sensibilidad predecía mejor que la propensión los miedos interpersonales, (2) la propensión predecía mejor que la sensibilidad los miedos SID, (3) los miedos a los animales y agorafóbicos eran similarmente predichos por ambas variables, y (4) el miedo a la evaluación negativa no era predicho por ninguna de las dos variables. Por otra parte, aunque tanto la propensión como la sensibilidad al asco predecían los síntomas totales de TOC, cuando se consideraban las subescalas de TOC por separado, las obsesiones de contaminación y compulsiones de lavado eran predichas por la propensión, mientras que los pensamientos obsesivos de daño a sí mismo/otros lo era por la sensibilidad al asco. Puede observarse que existen algunas similitudes entre nuestros resultados y los obtenidos por van Overveld y colaboradores, aunque también existen algunas diferencias que deben ser clarificadas con posteriores estudios. No obstante, tanto los datos referidos por van Overveld (2008) como obtenidos por nosotros (Sandín et al., 2008a) ponen de relieve que la propensión al asco y la sensibilidad al asco constituyen dos constructos separados que pueden relacionarse de forma diferente con las variables psicopatológicas.

\section{Sensibilidad al asco y estructura jerárquica de los miedos y las fobias}

Algunos autores han sugerido que la relación entre la sensibilidad al asco y los síntomas (miedos, etc.) y trastornos de ansiedad es un mero artefacto metodológico, o un epifenómeno, debido a la varianza que comparte la sensibilidad al asco con el rasgo de ansiedad o afecto negativo (p.ej., Kleinknecht et al., 1997; Muris et al., 1999; Thorpe y Salkovskis, 1998). En este sentido, la relación etiológica entre el asco y los trastornos de ansiedad que ha sido sugerida sería algo ilusorio, no sería una relación única sino una función de varianza compartida entre el asco y el rasgo de ansiedad. Otros autores (p.ej., Davey y Bond, 2006), sin embargo, han rechazado esta crítica, y defienden que el rasgo de ansiedad y la propensión al asco son constructos independientes, cada uno de los cuales tiene una relación única con los síntomas y trastornos de ansiedad. En apoyo de la hipótesis de Davey y Bond están los resultados de la mayor parte de la investigación llevada a cabo sobre el asco y los trastornos de ansiedad, en la que se han incluido medidas de rasgo de ansiedad (p.ej., Deacon y Olatunj, 2007; McDonald, Hartman y Vrana, 2008; Muris et al., 2008; Olatunji et al., 
2007d; Tolin et al., 2006; Sandín et al., 2008a; van Overveld, 2008). En estos estudios se ha demostrado que la asociación entre la propensión o sensibilidad al asco y los síntomas de ansiedad se mantiene (al menos para ciertos síntomas o trastornos) a pesar de haber sido controlado el efecto del rasgo del ansiedad (o neuroticismo o afecto negativo). La sensibilidad o propensión al asco parece mediar entre el rasgo de ansiedad y los síntomas de ansiedad (Olatunji et al., 2007d).

La propensión/sensibilidad al asco podría interpretarse como un factor de vulnerabilidad hacia los miedos/fobias. En este sentido, partiendo del modelo original de Taylor (1998) sobre la estructura jerárquica de las fobias, propusimos un modelo jerárquico sobre la etiología de los miedos y fobias basado en cuatro constructos (factores) de vulnerabilidad asociados a los cuatro principales tipos o factores de miedos (miedos a los animales, miedos SID, miedos sociales, y miedos situacionales o agorafóbicos) (véase Sandín, 1999; Sandín y Chorot, 1999). De acuerdo con este modelo, la estructura y etiología de los miedos y las fobias podría interpretarse desde cuatro factores de vulnerabilidad más o menos específicos denominados sensibilidad a la ansiedad (vulnerabilidad general para la mayoría de los miedos), sensibilidad al asco (vulnerabilidad a los miedos SID y a ciertos animales - animales relevantes al asco), sensibilidad a la evaluación social (vulnerabilidad a la fobia social), y sensibilidad al daño (vulnerabilidad a los miedos a ciertos animales - animales depredadores - y a miedos situacionales), y un factor más general (afecto negativo, neuroticismo, o rasgo de ansiedad; Sandín, 2003; Sandín et al., 1999). Del reciente estudio de McDonald et al. (2008) se desprende que, aunque tanto la sensibilidad al asco como el rasgo de ansiedad parecen contribuir independientemente en la explicación de la varianza de los cuatro tipos de miedos indicados, otros factores de vulnerabilidad deben estar necesariamente implicados (los autores identificaron un «factor general de miedo»). Estos resultados apoyan indirectamente nuestro modelo de vulnerabilidad de los miedos (Sandín y Chorot, 1999), pudiendo estar constituido este factor general de miedo posiblemente en su mayor par- te por la sensibilidad a la ansiedad. En nuestro reciente estudio (Sandín et al., 2008a) constatamos que la sensibilidad a la ansiedad estaba implicada básicamente en la predicción de todos los tipos de miedos y síntomas TOC (en contraste, la propensión y/o sensibilidad al asco y el afecto negativo se asociaban a síntomas más limitados). Esta especial relación entre la sensibilidad a la ansiedad y los miedos ha venido siendo señalada por nuestro grupo a través de diversos estudios (p.ej., Sandín, 1997; Sandín, Chorot, Valiente y Santed, 2002; Valiente, Sandín y Chorot, 2002a,b).

\section{COMENTARIOS FINALES}

Tras el análisis que hemos presentado en estas páginas parece claro que el asco (propensión y/o sensibilidad) se relaciona de forma consistente con algunos síntomas y trastornos de ansiedad, fundamentalmente con los miedos y fobias a ciertos animales (animales «repulsivos» y generalmente no peligrosos, tales como las arañas) y a estímulos relacionados con la sangre y el daño, así como también con el trastorno obsesivo-compulsivo. Esta relación se ha interpretado en términos de que el asco constituye un factor de vulnerabilidad, y está implicado en la etiología y mantenimiento de estos trastornos. Aunque en la última década se han llevado a cabo importantes estudios, y existe un cúmulo importante de datos al respecto, el conocimiento que se tiene actualmente sobre esta cuestión es únicamente preliminar. Por tanto, antes de finalizar con nuestra presentación, creemos necesario hacer algunas consideraciones sobre esta nueva frontera de investigación.

En un artículo reciente, Teachman (2006) se hacía eco de que aún no existe un claro acuerdo sobre como definir el asco patológico; ni tampoco sobre cómo influye el asco en la psicopatología. La definición del miedo patológico se apoya en la idea de que el miedo y la evitación sean excesivos e irracionales, dadas las características objetivas de la situación. No está claro, sin embargo, que pueda utilizarse un criterio similar para definir el asco patológico. Existen muchas circunstancias en las que los individuos sanos experimentan la amenaza de 
contaminación y asco sin que exista un peligro real, por lo que no parece que sea suficiente identificar el asco patológico en términos de reacciones irracionales. Aunque a este respecto aún no disponemos de unos criterios claros, como punto de partida para futuras investigaciones merece la pena tener en consideración los tres criterios siguientes sugeridos por Teachman (2006) para la delimitación del asco patológico: (1) La evaluación de la amenaza (p.ej., percepción de contaminación) o la respuesta al estímulo evocador debe ser excesiva (comparada con otras personas; no debe ser simplemente parte de una respuesta aceptada en una subcultura determinada). (2) La respuesta de asco debe causar malestar clínicamente significativo o perturbación, generalmente a través de conductas de evitación excesivas. Y (3) la simple información sobre la baja probabilidad de daño debido a la amenaza de contaminación (física, psicológica o moral) es insuficiente para reducir el malestar emocional o la evitación (creencias rígidas a pesar de la evidencia objetiva de lo contrario).

Si aún no conocemos a través de qué mecanismos influye el asco sobre los síntomas y trastornos de ansiedad aquí discutidos (aunque el grueso de la evidencia tiende a indicar que puede estar implicado en su etiología y mantenimiento, constituyendo un importante factor de riesgo), tampoco sabemos su alcance psicopatológico respecto a otros posibles problemas o trastornos. En concreto, hace exactamente diez años que Phillips et al. (1998) alertaron sobre la posibilidad de que el asco influyera en la psicopatología de muchos otros trastornos, aparte de ciertas fobias específicas. Estos autores sugirieron que el asco, por sus características y relación con la amenaza o miedo a la contaminación, debería en principio relacionarse con otras condiciones psiquiátricas como los trastornos obsesivo-compulsivos (como aquí hemos presentado, se ha producido un interés creciente sobre este tema en los últimos años), los trastornos alimentarios (por definición el asco se ha asociado básicamente a la comida), la fobia social (la sensibilidad al asco podría asociarse al temor al contacto físico con desconocidos), y las fobias situacionales y agorafobia (p.ej., por influencia de suciedad de los lugares públicos).
Aunque existe escasa investigación sobre la conexión del asco con otros trastornos, algunos estudios recientes han puesto de manifiesto que la sensibilidad al asco también parece asociarse a otros síntomas y/o trastornos psicológicos, entre los que se incluyen los trastornos alimentarios (Davey, Buckland, Tantow y Dallos, 1998; Troop, Treasure y Serpell, 2002; van Overveld, 2008), la hipocondría (Davey y Bond, 2006), diversas fobias situacionales, la fobia social y la agorafobia (Davey y Bond, 2006; Muris et al., 2008; Sandín et al., 2008a), y la fobia a vomitar (van Overveld, de Jong, Peters, van Hout y Bouman, 2008).

Si partimos de las peculiaridades que parece tener la emoción de asco, cabe suponer que la sensibilidad al asco podría estar implicada en un amplio abanico de problemas o trastornos psicológicos que la investigación futura deberá clarificar, entre los cuales, aparte de los aquí tratados, al menos merecería la pena resaltar los siguientes: trastornos somatoformes (GarcíaCampayo, Pascual, Alda y Oliván, 2005; Carmona, Pascual, Sánchez, Maldonado y Pereira, 2007), transexualismo y otros trastornos sexuales (Hurtado, Gómez y Donat, 2007; Olatunji y Sawchuk, 2005), trastornos alimentarios (Jorquera, Baños, Perpiña y Botella, 2005; Perpiñá, 1999), evitación interoceptiva (Sandín, 2005), sida y exclusión social (Ballester, 2005; Edo y Ballester, 2006; Flores, Borda y Sangregorio, 2006; Piña y Robles, 2005), cáncer y síntomas asociados a la quimioterapia (Méndez, 2005; Vega et al., 2004), fobias sociales y agorafobia (Bados, 2000; Botella y Gallardo, 1999; Echeburúa y de Corral, 1993; Gallego, Botella, Quero, Baños y García-Palacios, 2007; Sandín, Rodero, Santed y García-Campayo, 2006; Zubeidat, Sierra y Fernández-Parra, 2007), ansiedad de separación (Echeburúa, 1993; Méndez, Orgilés y Espada, 2008; Orgilés, Espada y Méndez, 2008; Sandín, 1997), y estrés postraumático (McNally, 2002; Talarn, Navarro, Rossel y Rigat, 2006).

Aunque existen diversas escalas de autoinforme validadas para medir la sensibilidad al asco, la mayor parte de ellas son estímulo-dependientes, lo cual supone que puede darse cierta confusión de medidas al relacionarlas con síntomas de ansiedad (p.ej., con miedos a los 
animales o a estímulos SID). Este problema es importante controlarlo, ya que de lo contrario estaríamos «inflando» de forma artificial las correlaciones entre asco y psicopatología. La reciente propuesta de van Overveld et al. (2006; van Overveld, 2008; Sandín et al., 2008a) basada en la introducción de una escala de propensión general, libre del efecto contextual, supone una solución al problema, aunque no invalida el uso de las escalas contextuales (necesarias para evaluar los dominios o áreas del asco). Así mismo, la propuesta de estos autores de separación conceptual de la propensión vs. sensibilidad del asco, abre una nueva frontera de investigación en el campo de la psicopatología del asco. Por otra parte, apenas se ha prestado interés a la dimensión de asco sociomoral (no está incluida en las escalas de evaluación al uso), ni a la contaminación mental (véase Herba y Rachman, 2007).

A nuestro juicio deberían mejorarse los procedimientos experimentales utilizados para estudiar las leyes mágicas del asco (leyes de contacto y similitud) y otros problemas en los que se emplean procedimientos de inducción de asco. Como ha sugerido McNally (2002), un problema con los métodos usados habitualmente para probar hipótesis sobre sensibilidad al asco es que no se consideran los incentivos que pueden contrarrestar el efecto de los estímulos de asco. Por ejemplo, un método común usado en la investigación puede consistir en pedir a los participantes que coman una galleta después de haber sido tocada por algún gusano. La cuestión es, ¿porqué alguien debe comer una galleta después de que un gusano se haya arrastrado sobre ella? La única razón para hacerlo es el hambre, o bien el afán de complacer al experimentador. Puesto que pocos sujetos experimentales suelen estar hambrientos, el hecho de comer galletas «contaminadas» en el laboratorio puede informarnos más sobre las tendencias de aquiescencia social que sobre la sensibilidad al asco.

Finalmente, el enfoque psicopatológico del presente artículo necesariamente nos lleva a considerar de qué manera podría beneficiarse la terapia de los trastornos aquí discutidos. En principio, lo que resulta claro es que incluso las fobias específicas no parece que se basen en emociones puras (p.ej., el miedo), algo que sue- le ocurrir también en otras emociones (Muela, García-León y Jiménez, 2007; Rueda y PérezGarcía, 2007). Más bien, parece que las fobias y otros trastornos de ansiedad pueden consistir en una combinación de emociones negativas, $\mathrm{y}$ en concreto, en algunos casos, en una combinación de miedo y asco. La implicación que esto pueda tener a la hora de llevar a cabo la terapia depende del papel que jueguen ambas emociones. Si el asco opera de modo similar al miedo, la distinción entre ambas emociones tendría escaso valor práctico (Woody y Teachman, 2000). Sin embargo, las diferencias que se han observado entre el asco y el miedo respecto a sus diversos componentes (subjetivo, cognitivo, fisiológico y conductual), así como las discrepancias en su adquisición y mantenimiento, sugieren que el manejo centrado en cada emoción (p.ej., induciendo respuestas de miedo o de asco) puede ser crítico para maximizar la eficiencia terapéutica. Por ejemplo, ignorar las respuestas de asco ante un determinado tratamiento de las fobias SID basado en la exposición (en vivo o imaginada), puede producir efectos negativos en el paciente e interferir en el proceso terapéutico. Lógicamente, para poder determinar qué tratamientos pueden beneficiarse manipulando o basándose en las respuestas de asco, depende del papel causal o funcional que tenga el asco en el trastorno específico. Si el asco está implicado en las creencias de contaminación o en los rituales compulsivos de un paciente con diagnóstico de TOC, el tratamiento podría mejorarse abordando específicamente el asco en el paciente (Berle y Phillips, 2006). Aunque las técnicas de exposición diseñadas para el tratamiento del miedo, también podrían, en ciertos casos, reducir los niveles de asco, no está tan claro que las intervenciones cognitivas del miedo (p.ej., las distintas formas de reestructuración cognitiva) puedan ser efectivas para reducir o suprimir el asco patológico, ya que la reevaluación cognitiva, por ejemplo de la ideación de contaminación, no es eficaz para modificar la evaluación del estímulo inductor de asco. Esta asincronía parece implicar que los tratamientos (o procedimientos) cognitivos utilizados para reducir el miedo pueden requerir cierto reajuste previo para que sean efectivos para el asco (Woody y Teachman, 2000). En 
cualquier caso, y aunque sólo nos encontremos en el principio del comienzo, la evidencia disponible apunta que el asco puede desempeñar un papel relevante en la etiopatogenia de determinados trastornos (fobias a los animales, fobias SID, TOC, etc.), por lo que el tratamiento de estos problemas necesariamente debería tener en cuenta este hecho y actuar en consecuencia. Todo sea para mejorar el actual arsenal de técnicas terapéuticas aplicadas al tratamiento de la ansiedad validadas empíricamente (véase Pelechano, 2007); y de paso para librarnos de caer en la fácil tentación de recurrir al empleo de las mal llamadas «terapias de tercera generación» (véase Öst, 2008).

\section{REFERENCIAS}

Bados, A. (2000). Agorafobia y ataques de pánico. Madrid: Pirámide.

Ballester, R. (2005). Aportaciones desde la psicología al tratamiento de las personas con infección por VIH/SIDA. Revista de Psicopatología y Psicología Clinica, 10, 53-69.

Belloch, A., Morillo, C., \& García-Soriano, G. (2006). Obsession subtypes: Relationships with obsessive-compulsive symptoms, dysfunctional beliefs and thought control strategies. Revista de Psicopatología y Psicología Clínica, 11, 65-78.

Berle, D., \& Phillips, E.S. (2006). Disgust and obsessivecompulsive disorder: An update. Psychiatry, 69, 228238.

Botella, C., y Gallardo, M. (1999). La fobia social. Madrid: Klinik.

Calder, A. J., Lawrence, A. D., \& Young, A. W. (2001). Neuropsychology of fear and loathing. Nature Reviews Neuroscience, 2, 352-363.

Carmona, J., Pascual, L. M., Sánchez, J. L., Maldonado, J. A., y Pereira, A. (2007). El papel de la amplificación somatosensorial en la predicción de síntomas respiratorios. Revista de Psicopatología y Psicología Clínica, 12, 15-22.

Cavanagh, K., \& Davey, G. C. L. (2000). The development of a measure of individual differences in disgust. Paper presented to the British Psychological Society, Winchester, UK.

Chorot, P., Sandín, B., Valiente, R.M., Santed, M. A., y Romero, M. (1997). Actitud hacia la enfermedad, ansiedad y sintomatología somática en pacientes con trastorno de pánico e hipocondría. Revista de Psicopatología y Psicología Clínica, 2, 123-136.
Davey, G. C. L. (1992). Characteristics of individuals with fear of spiders. Anxiety Research, 4, 299-314.

Davey, G. C. L. (1994). Self-reported fears to common indigenous animals in an adult UK population: The role of disgust sensitivity. British Journal of Clinical Psychology, 85, 541-554.

Davey, G. C. L., \& Bond, N. (2006). Using controlled comparisons in disgust psychopathology research: The case of disgust, hypochondriasis and health anxiety. Journal of Behavior Therapy and Experimental Psychiatry, 37, 4-15.

Davey, G. C. L., Buckland, G., Tantow, B., \& Dallos, R. (1998). Disgust and eating disorders. European Eating Disorders Review, 6, 201-211.

Davey, G. C. L., Forster, L., \& Mayhew, G. (1993). Familial resemblances in disgust sensitivity and animal phobias. Behaviour Research and Therapy, 31, 41-50.

Davey, G. C. L., McDonald, A. S., Hirisave, U., Prabhu, G. G., Iwawaki, S., Jim, C. I., Merckelbach, H., de Jong, P.J., Leung, P.W.L., \& Reimann, B.C. (1998). A crosscultural study of animal fears. Behaviour Research and Therapy, 36, 735-750.

de Jong, P. J., \& Merckelbach, H. (1998). Blood-injectioninjury phobia and fear of spiders: Domain specific individual differences in disgust sensitivity. Personality and Individual Differences, 24, 153-158.

de Jong, P. J., \& Peters, M. L. (2007). Blood-injection-injury fears: Harm- vs. disgust relevant selective outcome associations. Journal of Behavior Therapy and Experimental. Psychiatry, 38, 263-274.

de Jong, P. J., Andrea, H., \& Muris, P. (1997). Spider phobia in children: Disgust and fear before and after treatment. Behaviour Research and Therapy, 35, 559-562.

Deacon, B., \& Olatunji, B. O. (2007). Specificity of disgust sensitivity in the prediction of behavioural avoidance in contamination fear. Behaviour Research and Therapy, 45, 2110-2120.

Echeburúa, E. (1993). Trastornos de ansiedad en la infancia. Madrid: Pirámide.

Echeburúa, E., y de Corral, P. (1992). La agorafobia. Nuevas perspectivas de evaluación y tratamiento. Valencia: Promolibro.

Edo, M.T., y Ballester, R. (1996). Estado emocional y conducta de enfermedad en pacientes con VIH/SIDA y enfermos oncológicos. Revista de Psicopatología y Psicología Clínica, 11, 79-90.

Flores, E., Borda, M., y Sangregorio, M. A. (2006). SIDA y exclusión social: Alteraciones emocionales. Revista de Psicopatología y Psicología Clínica, 11, 37-49.

Gallego, M. J., Botella, C., Quero, S., Baños, R., y GarcíaPalacios, A. (2007). Propiedades psicométricas de la Escala de Miedo a la Evaluación Negativa versión breve (BFNE) en muestra clínica. Revista de Psicopatología y Psicología Clínica, 12, 163-176. 
García-Campayo, J., Pascual, A., Alda, M., y Oliván, A. (2005). Tratamiento psicológico en el trastorno de somatización: Eficacia y propuestas de intervención. Revista de Psicopatología y Psicología Clínica, 10, 15-23.

Haidt, J., McCauley, C., \& Rozin, P. (1994). Individual differences in sensitivity to disgust: A scale sampling seven domains of disgust elicitors. Personality and Individual Differences, 16, 701-713.

Herba, J. K., \& Rachman, S. (2007). Vulnerability to mental contamination. Behaviour Research and Therapy, 45 , 2804-2812.

Huijding, J., \& de Jong, P. J. (2007). Beyond fear and disgust: The role of (automatic) contamination-related associations in spider phobia. Journal of Behavior Therapy and Experimental Psychiatry, 38, 200-211.

Hurtado, F., Gómez, M., y Donat, F. (2007). Transexualismo y salud mental. Revista de Psicopatología y Psicología Clínica, 12, 43-58.

Jorquera, M., Baños, R.M., Perpiña, C., y Botella, C. (2005). La Escala de Estima Corporal (BES): Validación en una muestra española. Revista de Psicopatología y Psicología Clínica, 10, 173-192.

Kleinknecht, R. A., Kleinknecht, E. E., \& Thorndike, R. M. (1997). The role of disgust and fear in blood and injection-related fainting symptoms: A structural equation model. Behaviour Research and Therapy, 35, 1075-1087.

Levey, A. B., \& Martin, I. (1987). Evaluative conditioning: A case for hedonic transfer. In H.J. Eysenck \& I. Martin (Eds.), Theoretical foundations of behavior therapy (pp. 113-131). New York: Plenum Press.

Mancini, F., Gragnani, A., \& D’Olimpo, F. (2001). The connection between disgust and obsessions and compulsions in a non-clinical sample. Personality and Individual Differences, 31, 1173-1180.

Matchett, G., \& Davey, G. C. (1991). A test of a diseaseavoidance model of animal phobias. Behaviour Research \& Therapy, 29, 91-94.

Mayer B., Bos, A. E. R., Muris, P., Huijding, J., \& Vlielander, M. (2008). Does disgust enhance eating disorder symptoms? Eating Behaviors 9, 124-127.

McDonald, S. D., Hartman, N. S., \& Vrana, S. R. (2008). Trait anxiety, disgust sensitivity, and the hierarchic structure of fears. Journal of Anxiety Disorders, 22, 1059-1074.

McNally, R. (2002). Disgust has arrived. Journal of Anxiety Disorders, 16, 561-566.

Méndez, J. (2005). Psicooncología infantil: Situación actual y líneas de desarrollo futuras. Revista de Psicopatología y Psicología Clínica, 10, 33-52.

Méndez, X., Orgilés, M., y Espada, J. P. (2008). Ansiedad por separación: Psicopatología, evaluación y tratamiento. Madrid: Pirámide.

Merckelbach, H., de Jong, P.J., Arntz, A., \& Schouten, E. (1993). The role of evaluative learning and disgust sen- sitivity in the etiology and treatment of spider phobia. Advances in Behaviour Research and Therapy, 15, 243245.

Muela, J. A., García-León, A., y Jiménez, M. D. (2007). Relación de la esquizotipia psicométrica con variables emocionales y socioambientales. Revista de Psicopatología y Psicología Clínica, 12, 199-212.

Mulkens, S. A. N., de Jong, P. J., \& Merckelbach, H. (1996). Disgust and spider phobia. Journal of Abnormal Psychology, 105, 464-468.

Muris, P., Merckelbach, H., Nederkoorn, S., Rassin, I. C., Candel, I., \& Horselenberg, R. (2000). Disgust and psychopathological symptoms in a nonclinical sample. Personality \& Individual Differences, 29, 1163-1167

Muris, P., Merckelbach, H., Schmidt, H., \& Tierney, S. (1999). Disgust sensitivity, trait anxiety and anxiety disorders symptoms in normal children. Behaviour Research and Therapy, 37, 953-961.

Muris, P., van der Heiden, S., \& Rassin, E. (2008). Disgust sensitivity and psychopathological symptoms in nonclinical children. Journal of Behavior Therapy and Experimental Psychiatry, 39, 133-146.

Olatunji, B. O., \& Deacon, B. (2008). Specificity of disgust sensitivity in the prediction of fear and disgust responding to a brief spider exposure. Journal of Anxiety Disorders, 22, 328-336.

Olatunji, B. O., \& Sawchuk, C. N. (2005). Disgust: Characteristic features, social manifestations, and clinical implications. Journal of Social and Clinical Psychology, 24, 932-962.

Olatunji, B. O., Cisler, J. M., Deacon, B. J. Connolly, K., \& Lohr, J. M. (2007a). The Disgust Propensity and Sensitivity Scale-Revised: Psychometric properties and specificity in relation to anxiety disorder symptoms. Journal of Anxiety Disorders, 21, 918-930.

Olatunji, B. O., Haidt, J., McKay, D., \& David, B. (2008). Core, animal reminder, and contamination disgust: Three kinds of disgust with distinct personality, behavioural, physiological, and clinical correlates. Personality and Individual Differences, 42, 1243-1259.

Olatunji, B. O., Lohr, J. M., Sawchuk, C. N., \& Tolin, D. F. (2007b). Multimodal assessment of disgust in contamination-related obsessive compulsive disorder. Behaviour Research and Therapy, 45, 263-276.

Olatunji, B. O., Sawchuk, C. N., de Jong, P. J., \& Lohr, J. M. (2007c). Disgus sensitivity and anxiety disorder symptoms: Pychometric properties of the Disgust Emotion Scale. Journal of Psychopathology and Behavioral Assessment, 29, 115-124.

Olatunji, B. O., Sawchuk, C. N., Lohr, J.M., \& de Jong, P. J. (2004). Disgust domains in the prediction of contamination fear. Behaviour Research and Therapy, 42, 93-104.

Olatunji, B. O., Williams, N. L., Lohr, J. M., Connolly, K. M., Cisler, J., \& Meunier, S. A. (2007d). Structural dif- 
ferentiation of disgust from trait anxiety in the prediction of specific anxiety disorder symptoms. Behaviour Research and Therapy 45, 3002-3017.

Olatunji, B. O., Williams, N. L., Tolin, D. F., Abramowitz, J. S., Sawchuk, C. N., Lohr, J. M., \& Elwood, L.S. (2007e). The Disgust Scale: Item analysis, factor structure, and suggestions for refinement. Psychological Assessment, 19, 281-297.

Orgilés, M., Espada, J. P., y Méndez, X. (2008).Trastorno de ansiedad por separación en hijos de padres divorciados. Psicothema, 20, 383-388.

Öst, L. G. (2008). Efficacy of the third wave of behavioral therapies: A systematic review and meta-analysis. $\mathrm{Be}$ haviour Research and Therapy 46, 296-321

Page, A. C. (1994). Blood-injury phobia. Clinical Psychology Review, 14, 443-461.

Page, A. C. (2003). The role of disgust in faintness elicited by blood and injection stimuli. Journal of Anxiety Disorders, 17, 45-58.

Pelechano, V. (2007). Viejas y nuevas cuestiones en las viejas y nuevas terapias psicológicas. Revista de Psicopatología y Psicología Clínica, 12, 71-90.

Perpiñá, C. (1999). Trastornos alimentarios: Anorexia y bulimia. Madrid: Klinik.

Phillips, M. L., Senior, C., Fahy, T., \& David, A. S. (1998). Disgust-The forgotten emotion in psychiatry. British Journal of Psychiatry, 172, 373-375.

Phillips, M. L., Young, A. W., Senior, C., Brammer, M., Andrews, C., Calder, A.J., et al. (1997). A specific neural substrate for perceiving facial expressions of disgust. Nature, 389, 495-498.

Piña, J. A., y Robles, S. (2005). Psicología y VIH/SIDA en México: Su prevención con base en un modelo psicológico de salud biológica. Revista de Psicopatología y Psicología Clínica, 10, 71-83.

Rozin, P., \& Fallon, A. E. (1987). A perspective on disgust. Psychological Review, 94, 23-41.

Rozin, P., \& Nemeroff, C. (1990). The laws of sympathetic magic: A psychological analysis of similarity and contagion. En J.W. Stigler, R.A. Shweder \& G. Herd (Eds.), Cultural psychology: Essays on comparative human development (pp. 205-232). Cambridge: Cambridge University Press.

Rozin, P., Fallon, A. E., \& Mandell, R. (1984). Family resemblance in attitudes to food. Developmental Psychology, 20, 309-314.

Rozin, P., Haidt, J., \& McCauley, C. (2000). Disgust. In M. Lewis \& J. M. Haviland (Eds.), Handbook of emotions. New York: Guilford Press.

Rueda, B., y Pérez-García, A. M. (2007). Estudio de la alexitimia y de los procesos emocionales negativos en el ámbito de los factores de riesgo y la sintomatología cardiovascular. Revista de Psicopatología y Psicología Clínica, 12, 105-116.
Sandín, B. (1997). Ansiedad, miedos y fobias en niños y adolescentes. Madrid: Dykinson.

Sandín, B. (1999). Diagnóstico y fenomenología. En B. Sandín (Ed.), Las fobias específicas (pp. 43-55). Madrid: Klinik.

Sandín, B. (2003). Escalas PANAS de afecto positivo y negativo para niños y adolescentes (PANASN). Revista de Psicopatología y Psicología Clínica, 8, 173-182.

Sandín, B. (2005). Evitación interoceptiva: Nuevo constructo en el campo de los trastornos de ansiedad. Revista de Psicopatología y Psicología Clínica, 10, 103114.

Sandín, B., y Chorot, P. (1999). Etiología de las fobias. En B. Sandín (Ed.), Las fobias especificas (pp. 69-87). Madrid: Klinik.

Sandín, B., Chorot, P., Lostao, L., Joiner, T.E., Santed, M.A., y Valiente, R.M. (1999). Escalas PANAS de afecto positivo y negativo: Validación factorial y convergencia transcultural. Psicothema, 11, 37-51.

Sandín, B., Chorot, P., \& McNally, R.J. (2001). Anxiety sensitivity index: Normative data and its differentiation from trait anxiety. Behaviour Research and Therapy, 39, 213-219.

Sandín, B., Chorot, P., Olmedo, M., y Valiente, R. M. (2008a). Escala de Propensión y Sensibilidad al Asco-Revisada (DPSS-R): Propiedades psicométricas y relación del asco con los miedos y los síntomas obsesivo-compulsivos. Análisis y Modificación de Conducta, 34, 93-136.

Sandín, B., Chorot, P., Santed, M. A., y Valiente, R. M. (2002). Análisis factorial confirmatorio del Índice de Sensibilidad a la Ansiedad para Niños. Psicothema, 14, 333-339.

Sandín, B., Chorot, P., Valiente, R. M., y Santed, M. A. (1998). Frecuencia e intensidad de los miedos en los niños: Datos normativos. Revista de Psicopatología y Psicología Clínica, 3, 15-25.

Sandín, B., Chorot, P., Valiente, R. M., y Santed, M. A. (2002). Relación entre la sensibilidad a la ansiedad y el nivel de miedos en niños. Psicología Conductual, 10, 107-120.

Sandín, B., Chorot, P., Valiente, R. M., Santed, M. A., y Lostao, L. (2004). Dimensiones de sensibilidad a la ansiedad: Evidencia confirmatoria de la estructura jerárquica. Revista de Psicopatología y Psicología Clínica, 9, 19-33.

Sandín, B., Valiente, R. M., y Chorot, P. (1999). Material de apoyo para la evaluación de los miedos y fobias. En B. Sandín (Ed.), Las fobias específicas (pp. 161-188). Madrid: Klinik.

Sandín, B., Valiente, R. M., y Chorot, P. (2008b). Instrumentos para la evaluación de los miedos y las fobias. En B. Sandín (Ed.), Las fobias especificas (Ed. rev., pp. 165-205). Madrid: Klinik. 
Sandín, B., Valiente, R. M., Chorot, P., y Santed, M. A (2005). Propiedades psicométricas del Índice de Sensibilidad a la Ansiedad. Psicothema, 17, 478-483.

Sandín, B., Valiente, R. M., Chorot, P., y Santed, M. A. (2007a). ASI-3: Nueva escala para la evaluación de la sensibilidad a la ansiedad. Revista de Psicopatología y Psicología Clínica, 12, 91-104.

Sandín, B., Valiente, R. M., Chorot, P., y Santed, M. A. (2007b). Sensibilidad a la ansiedad versus rasgo de ansiedad en la predicción del nivel de miedos. Análisis y Modificación de Conducta, 33, 139-158.

Sawchuk, C. N., Lohr, J. M., Tolin, D. F., Lee, T. C. \& Kleinknecht, R. A. (2000). Disgust sensitivity and contamination fears in spider and blood-injection-injury phobias. Behaviour Research and Therapy, 38, 753-762.

Sawchuk, C. N., Lohr, J. M., Westendorf, D. H., Meunier, S. A., \& Tolin, D. F. (2002). Emotional responding to fearful and disgusting stimuli in specific phobics. Behaviour Research and Therapy, 40, 1031-1046.

Schienle, A., Schafer, A., Walter, B., Stark, R., \& Vaitl, V. (2005). Elevated disgust sensitivity in blood phobia. Cognition and Emotion, 19, 1229-1241.

Schienle, A., Stark, R., \& Vaitl, D. (2001). Evaluative conditioning: A possible explanation for the acquisition of disgust responses? Learning and Motivation, 32, 65-83.

Simpson, J., Carter, S., Anthony, S. H., \& Overton, P. G. (2006). Is disgust a homogeneous emotion? Motivation and Emotion, 30, 31-41.

Stark, R., Zimmermann, M., Kagerer, S., Schienle, A., Walter, B., Weygandt, M., \& Vaitl, D. (2007). Hemodynamic brain correlates of disgust and fear ratings. NeuroImage, 37, 663-673.

Talarn, A., Navarro, N., Rossel, L., y Rigat, A. (2006). Propuesta de especificadotes diagnósticos vinculados al estrés y el trauma: Una aportación a la nosología psicopatológica. Revista de Psicopatología y Psicología Clínica, 11, 107-114.

Tallis, F. (1996). Compulsive washing in the absence of phobic and illness anxiety. Behaviour Research and Therapy, 34, 361-362.

Taylor, S. (1998). The hierarchic structure of fears. Behaviour Research and Therapy, 36, 205-214.

Taylor, S. (Ed.) (1999). Anxiety sensitivity: Theory, research, and treatment of the fear of anxiety. Mahwah, NJ: LEA.

Teachman, B.A. (2006). Pathological disgust: In the thoughts, not the eye, of the beholder. Anxiety, Stress and Coping, 19, 335-351.

Thorpe, S. J., \& Salkovskis, P. M. (1998). Studies on the role of disgust in the acquisition and maintenance of specific phobias. Behaviour Research and Therapy, 36, 877-893.

Thorpe, S. J., Patel, S. P., \& Simonds, L. M. (2003). The relationship between disgust sensitivity, anxiety and obsessions. Behaviour Research and Therapy, 41, 13971409.

Tolin, D. F., Lohr, J. M., Sawchuk, C. N., \& Lee, T. C. (1997). Disgust and disgust sensitivity in blood-injection-injury and spider phobia. Behaviour Research and Therapy, 35, 949-953.

Tolin, D. F., Woods, C. M., \& Abramowitz, J. S. (2006). Disgust sensitivity and obsessive-compulsive symptoms in a non-clinical sample. Journal of Behavior Therapy and Experimental Psychiatry, 37, 30-40.

Tolin, D. F., Worhunsky, P., \& Maltby, N. (2004). Sympathetic magic in contamination related OCD. Journal of Behavior Therapy and Experimental Psychiatry, 35, 193-205.

Troop, N. A., Treasure, J. L., \& Serpell, L. (2002). A further exploration of disgust in eating disorders. European Eating Disorders Review, 10, 218.226.

Vaitl, D., Schienle, A., \& Stark, R. (2005). Neurobiology of fear and disgust. International Journal of Psychophysiology, 57, 1-4.

Valiente, R. M. (2001). Estructura y adquisición de los miedos en la infancia y la adolescencia. Madrid: Universidad Nacional de Educación a Distancia.

Valiente, R. M., Sandín, B., y Chorot, P. (2002a). Miedos comunes en niños y adolescentes: Relación con la sensibilidad a la ansiedad, el rasgo de ansiedad, la afectividad negativa y la depresión. Revista de Psicopatología y Psicología Clínica, 7, 61-70.

Valiente, R. M., Sandín, B., y Chorot, P. (2002b). Miedos comunes en niños y adolescentes: Su relación con la sensibilidad a la ansiedad y otras emociones negativas. Psiquis, 23, 217-225.

Valiente, R. M., Sandín, B., y Chorot, P. (2003). Miedos en la infancia y la adolescencia. Madrid: UNED.

Valiente, R. M., Sandín, B., Chorot, P., y Santed, M. A. (2004). Cuestionario español de miedos para niños FSSC-E. Ponencia. VII European Conference on Psychological Assessment. Benalmádena Costa (Málaga), 1-4 de abril.

Valiente, R. M., Sandín, B., Olmedo, M., Chorot, P., y Santed, M. A. (2008a). Spanish version of the Disgust ScaleRevised. Poster. XXIX International Congress of Psychology. Berlin (Alemania), 20-25 de julio.

Valiente, R. M., Sandín, B., Olmedo, M., Chorot, P., Santed, M.A., y Magaz, A. (2008b). Confirmatory factor analisys of the Disgust Scale-Revised. Poster. 38th EABCT Annual Congress. Helsinki (Finlandia), 10-13 de septiembre.

Valiente, R.M., Sandín, B., Chorot, P., y Tabar, A. (2003). Diferencias según la edad en la prevalencia e intensidad de los miedos durante la infancia y la adolescencia: Datos basados en el FSSC-R. Psicothema, 15, 414-419. van Overveld, M. (2008). Disgust in specific phobias: A dirt road to anxiety disorders. Maastrich: UPM. 
van Overveld, M., de Jong, P. J., \& Peters, M. L. (2006). Differential UCS expectancy bias in spider fearful individuals: Evidence toward an association between spiders and disgust-relevant outcomes. Journal of Behavior Therapy and Experimental Psychiatry, 37, 60-72. van Overveld, M., de Jong, P. J., Peters, M. L., Cavanagh, K., \& Davey, G. C. L. (2006). Disgust propensity and disgust sensitivity: separate constructs that are differentially related to specific fears. Personality and Individual Differences, 41, 1241-1252.

van Overveld, M., de Jong, P. J., Peters, M. L., van Hout, W. J. P. J., \& Bouman, T. K. (2008). An internet-based study of the relation between disgust sensitivity and emetophobia. Journal of Anxiety Disorders, 22, 524-531.

Vega, M. E., de Juan, A., García, A. López, J. M., López, C., López, A., Rivera, F., y Ramos, J. (2004). Aspectos psicológicos de la toxicidad de la quimioterapia. Psicooncología, 1, 137-150

Vernon, L. L., \& Berenbaum, H. (2002). Disgust and fear in response to spiders. Cognition and Emotion, 16, 809830.
Ware, J. W., Jain, K., Burgess, I., \& Davey, G. C. L. (1994). Disease-avoidance model: Factor analysis of common animal fears. Behaviour Research and Therapy, 32, 57-63.

Webb, K., \& Davey, G. C. L. (1992). Disgust sensitivity and fear of animals: Effect of exposure to violent or revulsive material. Anxiety, Stress and Coping, 5, 329-335.

Woody, S. R., \& Teachman, B. A. (2000). Intersection of disgust and fear: Normative and pathological views. Clinical Psychology: Science and Practice, 7, 291-311.

Woody, S. R., \& Tolin, D. F. (2002). The relationship between disgust sensitivity and avoidant behavior: Studies of clinical and nonclinical samples. Journal of Anxiety Disorders, 16, 543-559.

Woody, S. R., McLean, C., \& Klassen, T. (2005). Disgust as a motivator of avoidance ofspiders. Journal of Anxiety Disorders, 19, 461-475.

Zubeidat, I., Sierra, J. C., y Fernández-Parra, A. (2007). Subtipos de ansiedad social en población adulta e infantojuvenil: Distinción cuantitativa versus cualitativa. Revista de Psicopatología y Psicología Clínica, 12, 147-162. 